Check for updates

Cite this: RSC Adv., 2017, 7, 32336

Received 19th March 2017

Accepted 19th June 2017

DOI: $10.1039 / c 7 r a 03219 f$

rsc.li/rsc-advances

\section{Insects (Thrips hawaiiensis (Morgan)) change the stereochemical configuration of 1-phenylethanol emitted from tea (Camellia sinensis) flowers $\dagger$}

\begin{abstract}
Ying Zhou, $\stackrel{t}{t}^{\mathrm{a}}$ Lanting Zeng, $\stackrel{t}{\mathrm{t}}^{\mathrm{ab}}$ Yinyin Liao, ${ }^{\mathrm{a}}$ Fang Dong, ${ }^{\mathrm{c}}$ Qiyuan Peng, ${ }^{\text {ab }}$ Jianlong Li, ${ }^{\mathrm{d}}$ Jinchi Tang, ${ }^{d}$ Naoharu Watanabe ${ }^{\mathrm{e}}$ and Ziyin Yang (ID *ab

Insect attacks change the quantity of volatiles emitted from plants, but little is known about the effects on the volatiles' stereochemical configurations. 1-Phenylethanol (1PE) is a major internal volatile of tea (Camellia sinensis) flowers. In the present study, insect-damaged $C$. sinensis flowers in the field emitted a different $(R)-/(S)-1 \mathrm{PE}$ pattern than that emitted from undamaged flowers. Laboratory experiments showed that changes in the $(R)-/(S)-1 \mathrm{PE}$ ratio were caused by treatments with Thrips hawaiiensis (Morgan), a pest of cut flowers and vegetable crops. These treatments led to an increase in jasmonic acid (JA) through the activation of allene oxide synthase, which is involved in JA biosynthesis. Exposing flowers to exogenous JA induced an $(R)-/(S)-1 \mathrm{PE}$ emission pattern that was similar to that induced by a $T$. hawaiiensis attack, suggesting that JA was involved in the changes in the stereochemical configuration of 1PE emitted from the flowers after insect treatments. This study provides new insights into the involvement of volatiles in interactions between plants and insects, showing that not only the quantity but also the stereochemical configuration of plant volatiles is affected by insects.
\end{abstract}

\section{Introduction}

Plant volatile compounds are low molecular weight metabolites $(<300 \mathrm{Da})$ with relatively low boiling points $\left(<260^{\circ} \mathrm{C}\right)$ that can be emitted from flowers, leaves, fruits, and roots into the atmosphere or soil, allowing the plants to interact with other organisms. ${ }^{1}$ The volatiles emitted from plant vegetative parts are involved in interactions between plants and herbivores, and have several positive functions, including direct and indirect defenses against herbivores, and within-plant and plant-plant signaling. ${ }^{2,3}$ In general, herbivore attacks induce increases in the level of volatiles emitted from plant vegetative parts.

${ }^{a}$ Guangdong Provincial Key Laboratory of Applied Botany, Key Laboratory of South China Agricultural Plant Molecular Analysis and Genetic Improvement, South China Botanical Garden, Chinese Academy of Sciences, Xingke Road 723, Tianhe District, Guangzhou 510650, China. E-mail: zyyang@scbg.ac.cn; Tel: +86-20-38072989

${ }^{b}$ University of Chinese Academy of Sciences, No. 19A Yuquan Road, Beijing 100049, China

${ }^{c}$ Guangdong Food and Drug Vocational College, Longdongbei Road 321, Tianhe District, Guangzhou 510520, China

${ }^{d}$ Tea Research Institute, Guangdong Academy of Agricultural Sciences, Guangdong Provincial Key Laboratory of Tea Plant Resources Innovation and Utilization, Dafeng Road 6, Tianhe District, Guangzhou 510640, China

${ }^{e}$ Graduate School of Science and Technology, Shizuoka University, 3-5-1 Johoku, Nakaku, Hamamatsu 432-8561, Japan

$\dagger$ Electronic supplementary information (ESI) available. See DOI: 10.1039/c7ra03219f

\$ These authors contributed equally to the work.
However, less is known about changes in the composition of the volatiles induced by herbivore attack. Allmann and Baldwin reported that attacks by the specialist herbivore Manduca sexta induced a rapid $(Z) /(E)$ isomeric change in the green leaf volatiles released by Nicotiana plants. ${ }^{4}$ This change lowered the $(Z) /$ $(E)$ ratio of the green leaf volatiles blend, which increased the predation rate of predators that fed on the herbivore's eggs.

Floral volatiles are generally believed to play roles in attracting pollinators. In contrast to volatiles emitted from vegetative parts, floral volatiles are reduced markedly soon after pollination. ${ }^{5}$ Such quantitative postpollination changes in floral bouquets decreases the attractiveness of these flowers and increases the overall reproductive success of the plant by directing pollinators to unpollinated flowers. This is particularly important for plants with a low visitation rate, in which reproductive success is mostly pollinator limited. ${ }^{6}$ The direct effects of floral volatiles on pollinator attraction have been observed by many researchers. However, attracting pollinators may increase the probability of attracting herbivores, and thus, fragrance bouquets may also be defensive. Junker and Bluthgen demonstrated that floral volatiles repel potentially nectarthieving ants and suggested that floral volatiles may function as allomones against enemies, as well as synomones to attract mutualists. ${ }^{7}$ There is a growing appreciation for the indirect effects of floral volatiles, particularly for its unexpected impact on the visual discrimination by color-sensitive foragers, such as bumble bees and diurnal hawkmoths. ${ }^{8-10}$ Moreover, floral volatiles may play a key role as a selective attractant in plants 
with specialized pollination systems, particularly in cases where floral morphology does not function as a filter of flower visitors. ${ }^{11}$ The indirect effects of floral volatiles, including flower or fruit destruction and modified pollinator behavior, ${ }^{12}$ also result from their potential to attract floral enemies.

Insect visits/attacks change the quantity of volatiles emitted from either vegetative or floral plant parts, but less is known about the effects of insect visits/attacks on the stereochemical configurations of volatiles emitted from these parts. Our previous studies found that Camellia sinensis (tea) flowers contained phenylpropanoid/benzenoid compounds as their major internal volatiles, ${ }^{13-16}$ which are mostly involved in interactions between insects and flowers. 1-Phenylethanol (1PE) was identified as a major volatile phenylpropanoid/benzenoid compound in C. sinensis flowers. 1PE and its precursor acetophenone (AP) accounted for more than $60 \%$ of internal volatiles of $C$. sinensis flowers. ${ }^{13}$ In C. sinensis flowers, $1 \mathrm{PE}$ was the most major volatile compound with stereochemical configuration, while other volatiles with stereochemical configuration, for example linalool, occurred with little or trace amounts. Therefore, our study aimed to discover the mechanism of change of $(R)-/(S)-1 \mathrm{PE}$ ratio after insect attacks. In the present study, we found that an attack by Thrips hawaiiensis (Morgan) induced changes in the $(R)-/(S)-1 \mathrm{PE}$ ratio emitted from tea flowers. $T$. hawaiiensis attack led to an increase in jasmonic acid (JA). Moreover, exposing flowers to exogenous JA induced a $(R)-/(S)$ 1PE pattern that was similar to that emitted from flowers after an $T$. hawaiiensis attack. This study provides new insights into the involvement of volatiles in interactions between plants and insects, showing that not only the quantity but also the stereochemical configurations of plant volatiles could be affected by insects.

\section{Results and discussion}

T. hawaiiensis treatments induced significant changes in the $(R)-/(S)$-1PE ratio emitted from $C$. Sinensis flowers

To investigate the influences of insect attacks on the volatiles emitted by $C$. sinensis flowers, undamaged flowers and insectdamaged flowers (Fig. 1A) were collected from field experiments. The internal $1 \mathrm{PE}$ was reduced in insect-damaged flowers in contrast to undamaged flowers (Fig. 1B). However, insect attacks did not induce significant changes in the $(R)-/(S)$ ratio of the internal 1PE. Interestingly, after insect attacks, the flowers

\section{A}

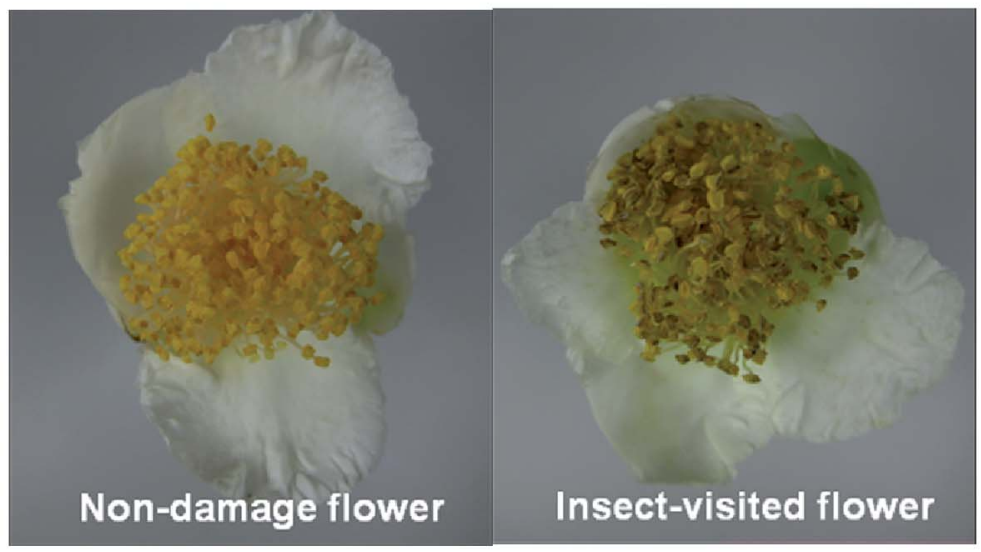

D

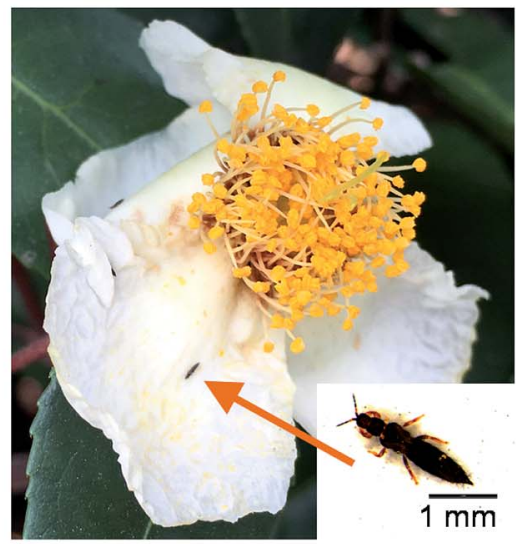

B

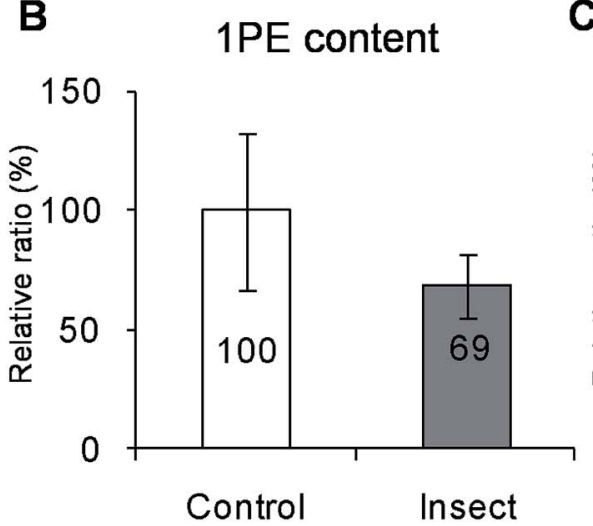

C

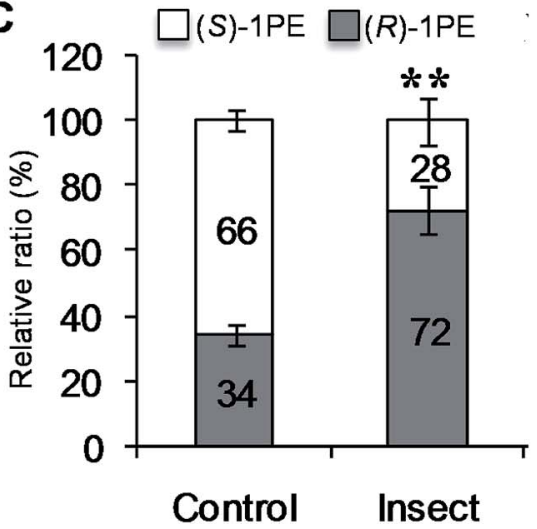

Fig. 1 Effect of insect attacks on phenotype and 1PE in C. sinensis flowers in field experiments. (A) The non-damage and insect-visited/damaged C. sinensis flowers. (B and C) Effects of insect attacks on internal 1PE content (B), and emitted (R)-/(S)-1PE ratio (C) in C. sinensis flowers. 1PE, 1phenylethanol. Control, undamaged flowers. Insect, insect-damaged flowers. (B) The concentration of $1 \mathrm{PE}$ in control was set as $100 \%$. (C) Significant differences between control and insect are indicated $(* * p \leq 0.01$ ). (D) Thrips hawaiiensis (Morgan) was found to be major insect that attacked $C$. sinensis flowers in tea filed. Data represent the mean value \pm standard deviation of three independent experiments performed in triplicate. 
emitted a different $(R)-/(S)-1 \mathrm{PE}$ pattern compared with that of undamaged flowers (control) (Fig. 1C).

While investigating which insect attacks induced changes in the $(R)-/(S)$-1PE ratio emitted from $C$. sinensis flowers, we found that $T$. hawaiiensis was the major insect attacking $C$. sinensis flowers in the field experiments (Fig. 1D). In laboratory experiments, adult $T$. hawaiiensis were used to treat $C$. sinensis flowers (Fig. 2A). After $6 \mathrm{~h}$, the $(R)-1 \mathrm{PE}$ level emitted from $C$. sinensis flowers increased (Fig. 2B), and the pattern was similar to that from insect-damaged flowers collected in the field experiments (Fig. 1C).

(A) Treatment of T. hawaiiensis on C. sinensis flowers. Control, undamaged flowers. Insect, T. hawaiiensis-damaged flowers. GCMS, gas chromatography-mass spectrometry; UPLC-QTOF-MS, ultra-performance liquid chromatography quadrupole-time-offlight mass spectrometry; SPME, solid-phase microextraction. (B) Effect of $T$. hawaiiensis attacks on emitted $(R)-/(R+S)-1 \mathrm{PE}$ ratio in $C$. sinensis flowers. 1PE, 1-phenylethanol. The ratio of $(R)-1 \mathrm{PE}$ to $(R+S)$-1PE in control was set as 1 . Significant differences between control and insect are indicated $(* p \leq 0.05)$. (C) UPLCQTOF-MS (negative) of phytohormones from authentic standards and extracts of $C$. sinensis flower. JA, jasmonic acid; ABA, abscisic acid; SA, salicylic acid. (D) Effect of T. hawaiiensis attacks on phytohormones contents in $C$. sinensis flowers. The contents of JA, ABA, and SA were calculated based on peak area of $\mathrm{m} / \mathrm{z}$ $209.118\left([\mathrm{M}-\mathrm{H}]^{-}\right.$of JA), $m / z 263.128\left([\mathrm{M}-\mathrm{H}]^{-}\right.$of ABA $)$, and $m / z$ $137.024\left([\mathrm{M}-\mathrm{H}]^{-}\right.$of SA), respectively. The concentrations of phytohormones in control were set as 1 . (E) Treatment of $C$. sinensis flowers using JA. JA, jasmonic acid. JA, $0.5 \mathrm{mM}$ jasmonic acid treatment. (F) Effects of jasmonic acid treatment on emitted $(R)-/(R+S)-1 \mathrm{PE}$ in $C$. sinensis flowers. The ratio of $(R)-1 \mathrm{PE}$ to $(R+$ $S)-1 \mathrm{PE}$ in control was set as 1 . Significant differences between control and JA are indicated (**p $\leq 0.01)$. Data represent the mean value \pm standard deviation of three independent experiments performed in triplicate.

In nature, floral structures, colors, and volatiles are critical factors in interactions between insects and flowers. Although floral structure and color provide important information for insects at a close range, floral volatiles can function as both longand short-distance attractants and as a nectar guide to a variety of insects. ${ }^{18}$ The pollination of flowers by insects leads to changes in scent emission. These changes in scent emission after pollination include a general decrease in all of the scent compounds, as found in Platanthera bifolia (Orchidaceae), ${ }^{19}$ or a decrease in some compounds, while others increase or remain unchanged, as was discovered in Ophrys orchids. ${ }^{20}$ However, it has not been reported that the stereochemical configurations of floral volatiles could be affected by insects, and there is only one report on vegetative volatiles. Allmann et al. reported that attacks by the specialist herbivore $M$. sexta larvae and the addition of their oral secretions to mechanical wounds induced a rapid $(Z) /(E)$ isomeric change in the green leaf volatiles released from Nicotiana plants. ${ }^{4}$ This change lowered the $(Z) /(E)$ ratio of the green leaf volatiles blend, resulting in an increase in the predation rate of predators that fed on the herbivore's eggs. These findings suggest that, in some cases, insect responses are dependent on the content of the volatile emission rather than merely the quantity. Our study

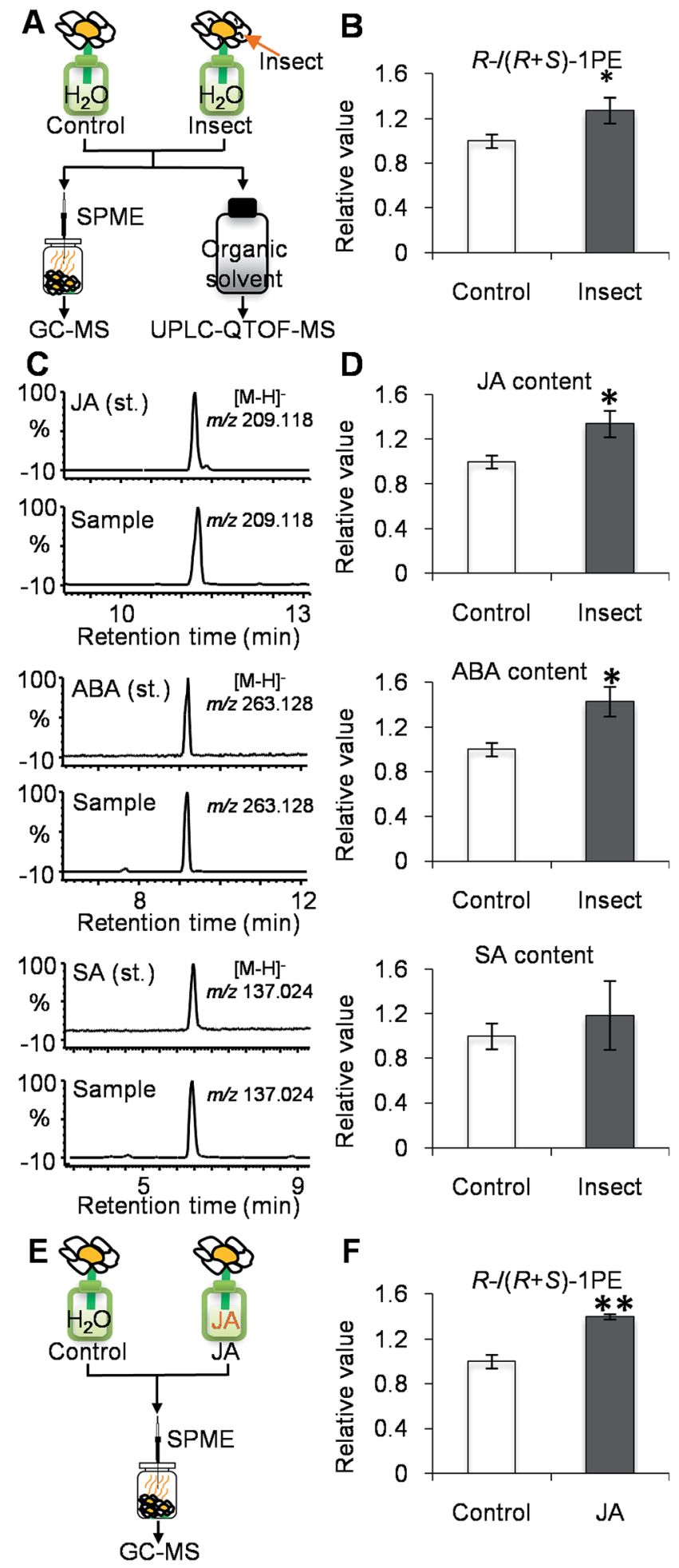

Fig. 2 Effect of Thrips hawaiiensis (Morgan) attacks on phytohormones and emitted $(R)-/(S)-1 \mathrm{PE}$ ratio in $C$. sinensis flowers in lab experiments.

indicated that such insect-induced changes in the stereochemical configuration of the volatiles occurred in not only vegetative parts but also in floral parts. Further studies on the effects of changes in the stereochemical configurations of floral volatiles on insects will help us understand the need for these flower responses. 
JA was involved in changes in the $(R)-/(S)-1 P E$ ratio emitted from $C$. sinensis flowers induced by $T$. hawaiiensis treatment To investigate if phytohormones are involved in changes in the $(R)-/(S)$-1PE ratio emitted from $C$. sinensis flowers induced by $T$. hawaiiensis treatments, we first analyzed the levels of phytohormones, including JA, abscisic acid (ABA), and salicylic acid, in C. sinensis flowers $6 \mathrm{~h}$ after a $T$. hawaiiensis treatment. Using the UPLC-QTOF-MS technique, the three
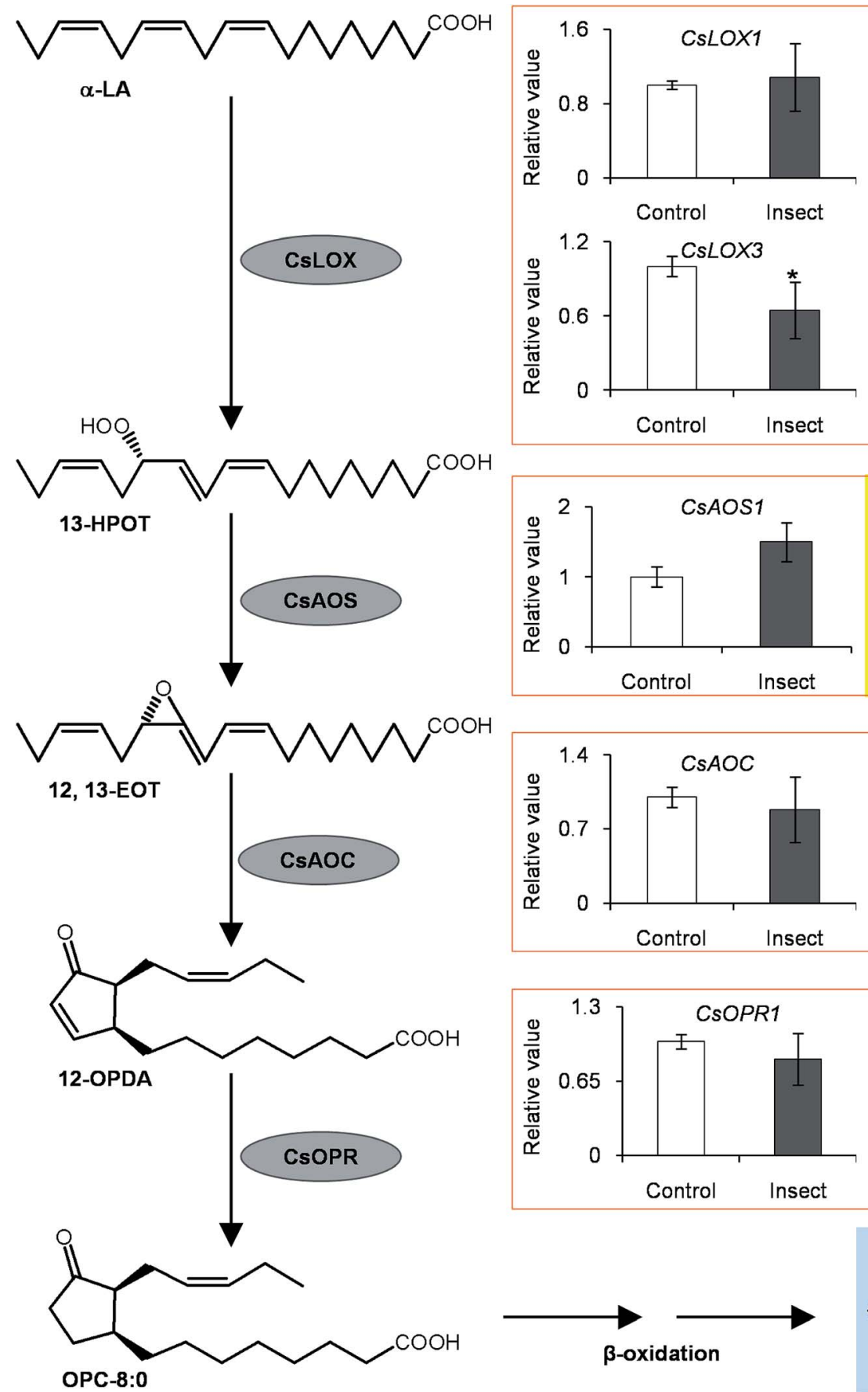
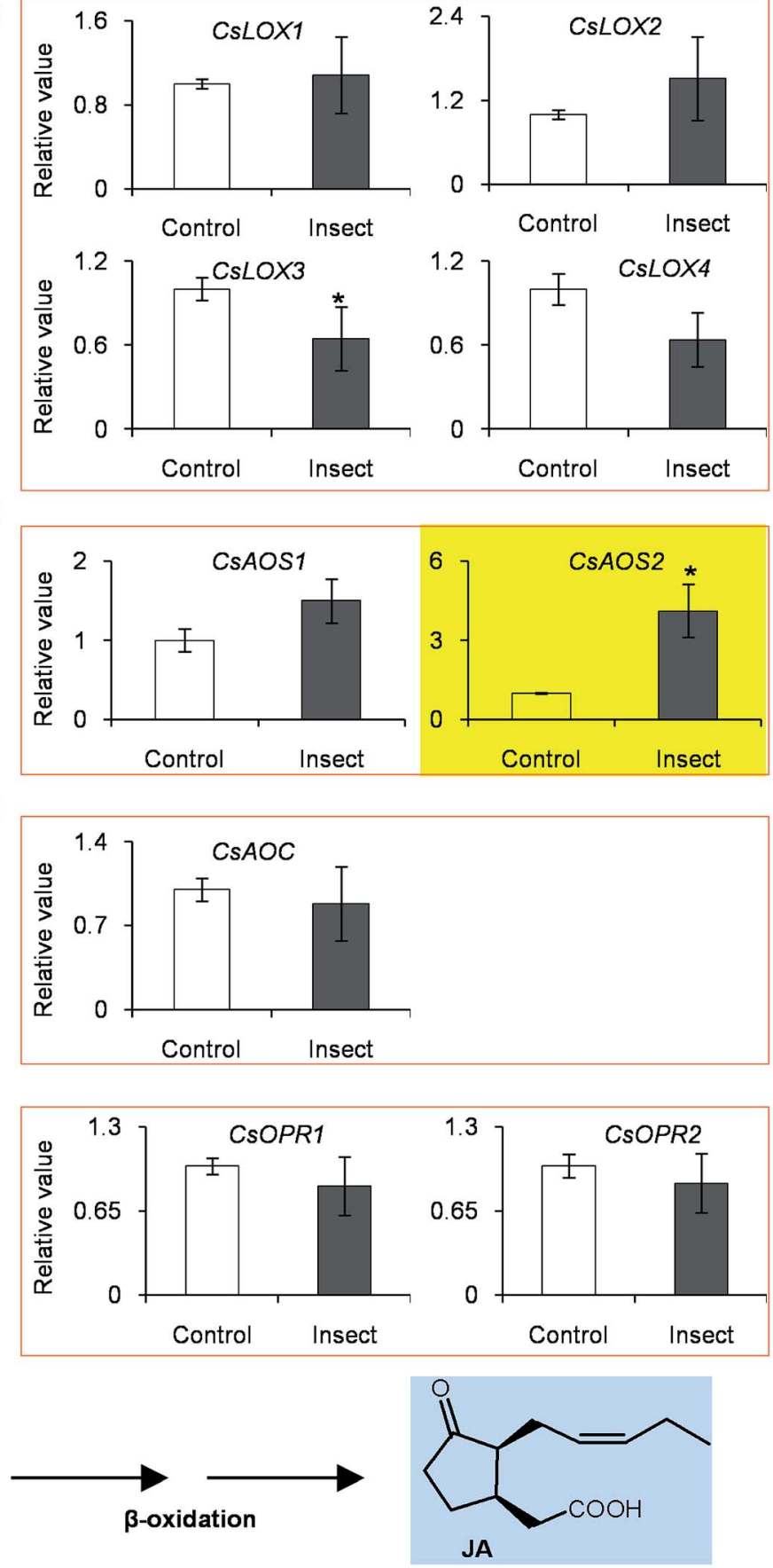

Fig. 3 Effect of Thrips hawaiiensis (Morgan) attacks on expression levels of jasmonic acid synthesis related genes in C. sinensis flowers. AOC, allene oxide cyclase; AOS, allene oxide synthase; 12,13-EOT, 12,13(S)-epoxy-9(Z),11,15(Z)-octa-decatrienoic acid; 13-HPOT, 13(S)-hydroperoxy linolenic acid; JA, jasmonic acid; LA, linolenic acid; LOX, 9/13-lipoxygenase; OPC-8:0; 3-oxo-2[2' (Z)-pentenyl]-cyclopentane-1-octanoic acid; 12-OPDA, 12-oxo-phytodienoic acid; OPR, 12-oxo-phytodienoic acid reductase. Control, undamaged flowers. Insect, T. hawaiiensis -damaged flowers. Transcript abundance was calculated based on the difference in cycle threshold $\left(C_{t}\right)$ values between target gene and internal reference gene transcripts by the normalized relative quantitation $2^{-\Delta \Delta C_{t}}$ method. The expression level in control was set as 1 . Significant differences between control and insect are indicated $(* p \leq 0.05)$. Data represent the mean value \pm standard deviation of three independent experiments performed in triplicate. 
phytohormones were detected in C. sinensis flowers (Fig. 2C). The JA and ABA contents were significantly increased $6 \mathrm{~h}$ after the T. hawaiiensis treatment, while the SA content did not show a significant change (Fig. 2D). To further investigate if the phytohormones were involved in changes in the $(R)-/(S)$ $1 \mathrm{PE}$ ratio emitted from $C$. sinensis flowers induced by the $T$. hawaiiensis treatment, JA and ABA, which were increased by the T. hawaiiensis treatment (Fig. 2D), were independently supplied to $C$. sinensis flowers. The exogenous ABA treatment did not significantly affect the $(R)-/(S)-1 \mathrm{PE}$ ratio emitted from flowers (Fig. S1 $\dagger$ ), but the exogenous application of JA to the flowers induced an increase in the level of $(R)-1 \mathrm{PE}$, resulting in a $(R)-/(S)-1 \mathrm{PE}$ pattern that was similar to that emitted from the insect-damaged flowers (Fig. $2 \mathrm{E}$ and F). We also investigated whether ethylene, which is generally reported to be involved in insect and floral volatiles, affected the $(R)-/(S)-1 \mathrm{PE}$ ratio emitted from flowers. Like the ABA treatments, ethylene did not significantly affect the $(R)-/(S)-1 \mathrm{PE}$ ratio emitted from flowers (Fig. S1 $\dagger$ ). Thus, JA was involved in the altered $(R)-/(S)$ $1 \mathrm{PE}$ ratio emitted from $C$. sinensis flowers induced by the $T$. hawaiiensis treatments. We also investigated the effects of insect treatments on the expression levels of genes involved in JA biosynthesis, including 9/13-lipoxygenase, allene oxide synthase, allene oxide cyclase, 12-oxo-phytodienoic acid reductase. Only the $C$. sinensis allene oxide synthase 2 gene's expression was significantly activated $6 \mathrm{~h}$ after the $T$. hawaiiensis treatment (Fig. 3).

To investigate how $T$. hawaiiensis treatments affected the $(R)-/(S)-1 \mathrm{PE}$ ratio emitted from C. sinensis flowers, we investigated the effects of $T$. hawaiiensis treatments on the formation of 1PE pathways. There are two pathways for the formation of $1 \mathrm{PE}$. One pathway leads from the glycosides of $1 \mathrm{PE}$ to $1 \mathrm{PE} .{ }^{14} 1 \mathrm{PE}$ $\beta$-primeverosides are the main glycosides of $1 \mathrm{PE}$. $\beta$-primeverosidase is involved in the hydrolysis of $1 \mathrm{PE} \beta$-primeverosides to form free $1 \mathrm{PE}$, while glycosyl transferases are responsible for the transformation from free $1 \mathrm{PE}$ to $1 \mathrm{PE} \beta$-primeverosides (Fig. 4A). ${ }^{14,21,22}$ The $(R) /(S)-1 \mathrm{PE} \quad \beta$-primeveroside ratio was not affected by T. hawaiiensis treatments (Fig. 4B). In addition, the $\beta$-primeverosidase (CsPri) and glycosyl transferases (CsGT1 and CsGT2) expression levels were not significantly increased by $T$. hawaiiensis treatments (Fig. 4C-E). The other pathway leads from acetophenone to $1 \mathrm{PE} .{ }^{13}$ Because the enzymes in this pathway are still unknown in plants, we used stable isotopelabeling acetophenone as a supplement given to the flowers. Then, T. hawaiiensis was co-treated with the $\left[{ }^{2} \mathrm{H}_{5}\right] \mathrm{AP}$ supplement (Fig. 5A). The emitted $\left[{ }^{2} \mathrm{H}_{5}\right] 1 \mathrm{PE}$ was collected, and $(R)-$ $\left[{ }^{2} \mathrm{H}_{5}\right] 1 \mathrm{PE}$ was increased by $T$. hawaiiensis attack (Fig. 5B), suggesting that the $T$. hawaiiensis treatment mainly affected the pathway leading from AP to $1 \mathrm{PE}$, thus leading to a change in the emitted $(R) /(S)$-1PE ratio.

Phytohormones are key links between insects and the formation of plant volatiles. JA is mostly reported to be involved in interactions between insects and volatiles from plant vegetative parts, while ethylene is mainly responsible for interactions between insects and volatiles from plant floral parts. There is considerable evidence that many postpollination processes in flowers, such as corolla abscission and/or
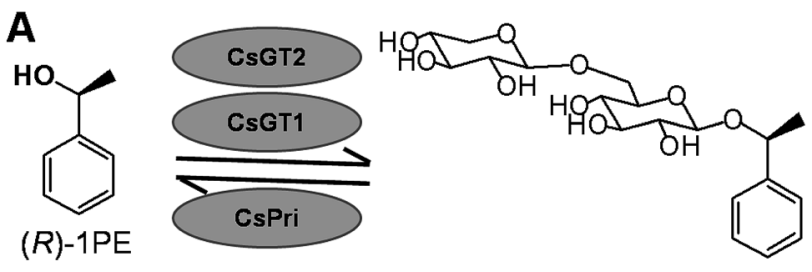

(R)-1PE-Pri

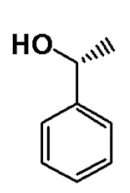

(S)-1PE

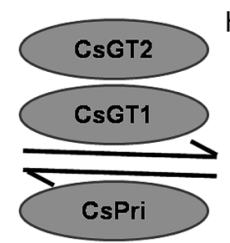

B
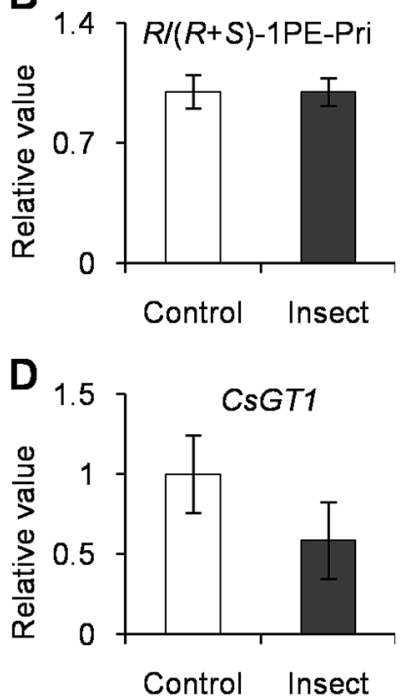

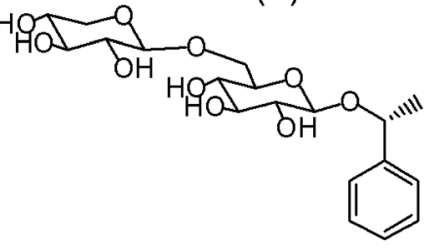

(S)-1PE-Pri
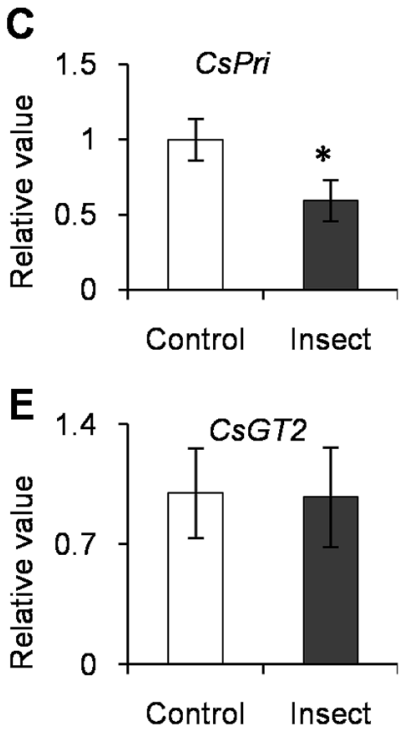

Fig. 4 Effect of Thrips hawaiiensis (Morgan) attacks on $(R)-/(R+S)$ $1 \mathrm{PE}-$ Pri ratio and expression levels of CSGT1, CsGT2, and CSPri in C. sinensis flowers. (A) Schemes of transformation between $(R)-/(S)-1 P E-$ Pri and $(R)-/(S)-1 \mathrm{PE}$. 1PE, 1-phenylethanol; 1PE-Pri, 1PE- $\beta$-primeveroside; GT, glycosyltransferases; Pri, $\beta$-primeverosidase. (B) Effect of $T$. hawaiiensis attacks on $(R)-/(R+S)-1 \mathrm{PE}-$ Pri in $C$. sinensis flowers. Control, undamaged flowers. Insect, $T$. hawaiiensis-damaged flowers. The ratio of $(R)$-1PE-Pri to $(R+S)$-1PE-Pri in control was set as 1. (C-E) Effect of T. hawaiiensis attacks on expression levels of CsPri, CSGT1, and CSGT2 in C. sinensis flowers. Transcript abundance was calculated based on the difference in cycle threshold $\left(C_{t}\right)$ values between target gene and internal reference gene transcripts by the normalized relative quantitation $2^{-\Delta \Delta C_{t}}$ method. The expression level in control was set as 1. Significant differences between control and insect are indicated $(* p$ $\leq 0.05$ ). Data represent the mean value \pm standard deviation of three independent experiments performed in triplicate.

senescence, pigmentation changes in the perianth, and ovary maturation, are mediated by endogenous ethylene that is produced as a consequence of pollination. ${ }^{23-25}$ In petunia, pollination leads to an increase in ethylene synthesis. ${ }^{5}$ Pollination also affects floral scent emission. ${ }^{5}$ Moreover, there is growing evidence that in many cases, plant responses to ethylene are associated with changes in gene expression. ${ }^{5,26}$ In the present study, change in the $(R)-/(S)$-1PE ratio emitted from flowers was not due to ethylene (Fig. S1†), but resulted from JA 

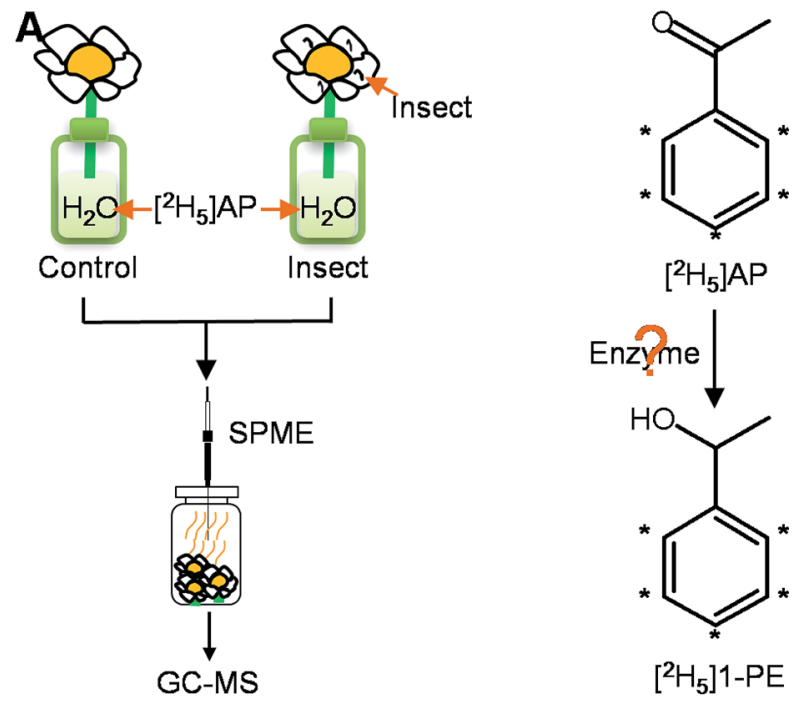

B

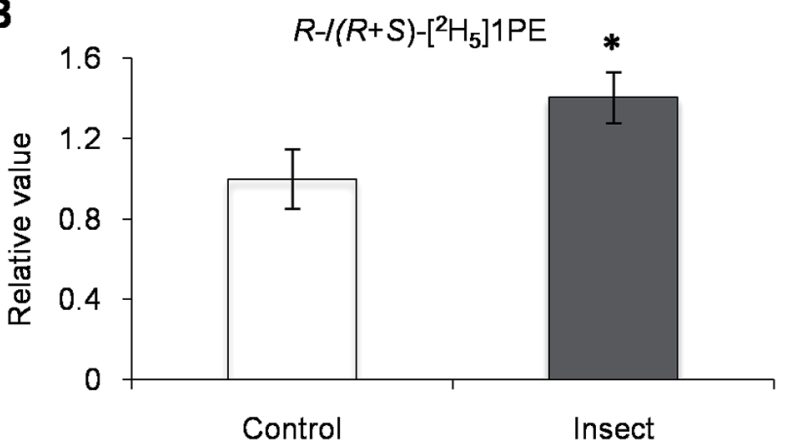

Fig. 5 Effect of Thrips hawaiiensis (Morgan) attacks on conversion of emitted labelled 1PE from labelled AP in C. sinensis flowers. (A) Treatment of $C$. sinensis flowers using insect. Control, undamaged flowers. Insect, T. hawaiiensis-damaged flowers. AP, acetophenone; GC-MS, gas chromatography-mass spectrometry; 1PE, 1-phenylethanol; SPME, solid-phase microextraction. (B) Effect of $T$. hawaiiensis attacks on emitted $(R)-/(R+S)-\left[{ }^{2} \mathrm{H}_{5}\right] 1 \mathrm{PE}$ in $\mathrm{C}$. sinensis flowers. Control, undamaged flowers. Insect, $T$. hawaiiensis-damaged flowers. The ratio of $(R)-\left[{ }^{2} \mathrm{H}_{5}\right] 1 \mathrm{PE}$ to $(R+S)-\left[{ }^{2} \mathrm{H}_{5}\right] 1 \mathrm{PE}$ in control was set as 1 . Significant differences between control and insect are indicated $\left({ }^{*} p \leq\right.$ $0.05)$. Data represent the mean value \pm standard deviation of three independent experiments performed in triplicate.

(Fig. 2F), suggesting that JA is another important phytohormone involved in volatile-mediated interactions between flowers and insects.

\section{Conclusions}

Insect attacks can lead to changes in the quantities of emitted plant volatiles, but the present study provides evidence that the stereochemical configurations of plant volatiles are also affected by insects. Moreover, JA, which is generally involved in relationships between insects and volatiles from vegetative plant parts, was found to also be responsible for changes in the stereochemical configuration of floral volatiles induced by insects (Fig. 6). The results obtained from this study will advance our understanding of the interactions between insects and plants mediated by volatiles.

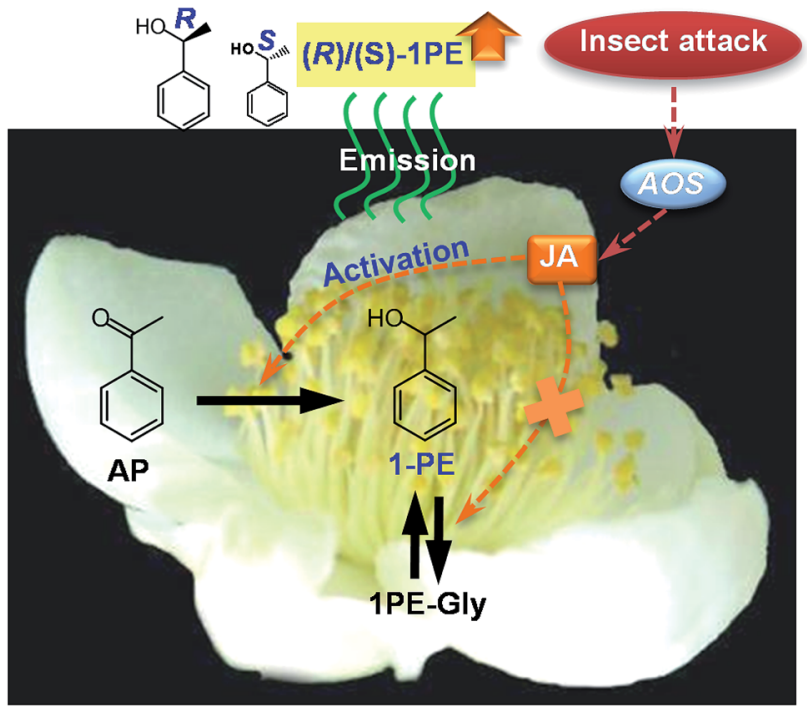

Fig. 6 Proposed schematic model of change in ratio of (R)-1PE to (S)$1 P E$ emitted from $C$. sinensis flowers exposed to insect attacks. AP, acetophenone; 1PE, 1-phenylethanol; 1PE-Gly, glycosides of 1-phenylethanol; JA, jasmonic acid; AOS, allene oxide synthase.

\section{Materials and methods}

\section{Chemicals}

ABA was purchased from Aladdin Industrial Co. Shanghai, China. $\left[{ }^{2} \mathrm{H}_{5}\right]$ AP was purchased from Cambridge Isotope Laboratories Inc., Cambridge, MA. Amberlite XAD-2 column and polyvinylpolypyrrolidone (PVPP) were purchased from SigmaAldrich Co. Ltd., USA. Ethephon was purchased from Fluka Analytical, Shanghai, China. Ethyl $n$-decanoate, $1 \mathrm{PE},(R)-1 \mathrm{PE}$ and $(S)$-1PE were purchased from Wako Pure Chemical Industries Ltd., Japan. Formic acid was purchased from CNW Technologies, Germany. JA was purchased from Tokyo Chemical Industry Co., Ltd., Japan. Methanol was purchased from EMD Millipore Corporation, Germany. $N$-Methyl- $N$-(trimethylsilyl)trifluoroacetamide (MSTFA) was purchased from Regis Chemical Co., USA. Quick RNA Isolation Kit was purchased from Huayueyang Biotechnology Co., Ltd., China. $2 \times$ SYBR Green Universal PCR Mastermix were purchased from Bio-Rad Laboratories, USA.

\section{Plants and treatments}

The nearly open flowers of $C$. sinensis var. Jinxuan were obtained from tea fields at the South China Agricultural University (Guangzhou, China) with stalks. The harvested flowers were reared in the laboratory to prevent insect attack for $16 \mathrm{~h}$ until fully-opened. T. hawaiiensis is a major pest founded in C. sinensis flowers in tea fields at the South China Agricultural University. They damage the flowers and suck the juice with rasping-mouthparts. In the laboratory experiments, the following three treatments were used: (1) insect treatments: five insects (adult T. hawaiiensis) were placed on a fully-opened flower that was in water. After treatment, the flowers were checked to make sure that the insects existed. The control was 
an individual fully-opened flower that was in water. (2) Phytohormone treatments: the individual fully-opened flower was placed in a solution containing $0.5 \mathrm{mM} \mathrm{JA}$, ABA, ethylene. The control was an individual fully-opened flower that was in water. (3) Stable labeling $\left[{ }^{2} \mathrm{H}_{5}\right] \mathrm{AP}$, the precursor of $1 \mathrm{PE},{ }^{\mathbf{1 3}}$ feeding treatment: five insects (adult $T$. hawaiiensis) were placed on a fully-opened flower that was in the $12 \mathrm{mM}\left[{ }^{2} \mathrm{H}_{5}\right] \mathrm{AP}$ solution. The control was an individual fully-opened flower that was in the $12 \mathrm{mM}\left[{ }^{2} \mathrm{H}_{5}\right] \mathrm{AP}$ solution. All of the treatments were performed for $6 \mathrm{~h}$ at room temperature. Every three flowers were used as one replicate for the collection of volatiles emitted from the flowers. Every 10 flowers were collected as one replicate for metabolite, phytohormone, and gene expression analyses. Each treatment was performed in triplicate.

\section{Extraction of internal volatiles and collection of flower- emitted volatiles}

To extract internal volatiles, $0.3 \mathrm{~g}$ finely powdered flowers was extracted with $2 \mathrm{~mL} \mathrm{CH}_{2} \mathrm{Cl}_{2}$ containing $5 \mathrm{nmol}$ ethyl $n$-decanoate as an internal standard. The extract was dried over anhydrous sodium sulfate. Then, $1 \mu \mathrm{L}$ of the extract was subjected to a gas chromatography-mass spectrometry (GC-MS) analysis. To collect the emitted volatile of flowers, $250 \mathrm{~mL}$ beaker which contained three flowers were sealed with silver paper. The solid phase microextraction (divinylbenzene/ carboxen/polydimethylsiloxane) was inserted into the beaker and the volatiles emitted from the flowers were collected by solid phase microextraction for $1 \mathrm{~h}$ at room temperature. The volatiles absorbed by the solid phase microextraction were analyzed using GC-MS.

\section{Analysis of 1PE, $(R)-1 \mathrm{PE}$, and $(S)$-1PE by GC-MS}

Samples were analyzed using GC-MS equipped with a SUPELCOWAX ${ }^{\mathrm{TM}} 10$ column (Supelco Inc., $30 \mathrm{~m} \times 0.25 \mathrm{~mm} \times$ $0.25 \mu \mathrm{m})$. The injector temperature was $240{ }^{\circ} \mathrm{C}$, the splitless mode was used with a splitless time of $1 \mathrm{~min}$, and helium was the carrier gas at a flow rate of $1.0 \mathrm{~mL} \mathrm{~min}^{-1}$ throughout the program. The column was used at an initial temperature of $40{ }^{\circ} \mathrm{C}$, a ramp of $5^{\circ} \mathrm{C} \mathrm{min}{ }^{-1}$ to $250{ }^{\circ} \mathrm{C}$, and then a hold at $250{ }^{\circ} \mathrm{C}$ for $15 \mathrm{~min}$. The MS analyses were carried out in full scan (mass range $m / z 70-200$ ) and selected ion-monitoring mode (SIM) (for 1PE: $m / z 77,79$, and 107). 1PE was identified by comparing their retention times and mass spectra with those of authentic compounds.

To determine the stereochemistry of $1 \mathrm{PE}[(R)-1 \mathrm{PE}$, and $(S)$ 1PE], a GC-MS equipped with an InertCap ${ }^{\mathrm{TM}}$ CHIRAMIX column (GL Sciences, $30 \mathrm{~m} \times 0.25 \mathrm{~mm} \times 0.25 \mu \mathrm{m}$ ) was employed. The injector temperature was $180{ }^{\circ} \mathrm{C}$, the splitless mode was used with a splitless time of $1 \mathrm{~min}$, and helium was the carrier gas at

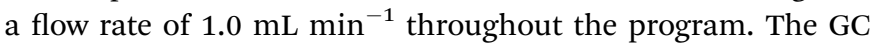
temperatures were $60{ }^{\circ} \mathrm{C}$ for $2 \mathrm{~min}$, ramp of $40^{\circ} \mathrm{C} \mathrm{min}^{-1}$ to $105{ }^{\circ} \mathrm{C}$, followed by $2^{\circ} \mathrm{C} \min ^{-1}$ to $137{ }^{\circ} \mathrm{C}$, then $80^{\circ} \mathrm{C} \mathrm{min}^{-1}$ to $180{ }^{\circ} \mathrm{C}$, and finally $180{ }^{\circ} \mathrm{C}$ for $10 \mathrm{~min}$. The MS was operated in SIM mode [for $(R)$ or $(S)-1 \mathrm{PE}: m / z 77,79,107$, and 122; for $\left[{ }^{2} \mathrm{H}_{5}\right]$ $(R)$ or $(S)$-1PE: $m / z$ 82, 84, 112, and 127]. (R)-1PE and $(S)$-1PE were identified by comparing their retention times and mass spectra with those of authentic compounds.

\section{Extraction and analysis of glycosides of 1PE}

The methods used to extract and analyze the glycosides of 1PE were described previously. ${ }^{14}$ Briefly, $1 \mathrm{~g}$ of finely powdered flower was extracted with $6 \mathrm{~mL}$ methanol by vortexing for $30 \mathrm{~s}$, followed by an ultrasonic extraction for $10 \mathrm{~min}$. The extracts were mixed with $6 \mathrm{~mL} \mathrm{CHCl}_{3}$ and $2.4 \mathrm{~mL} \mathrm{H}_{2} \mathrm{O}$ for phase separation. After centrifugation for $5 \mathrm{~min}$ at $3500 \mathrm{~g}$, the upper phase was transferred to a new tube and dried under vacuum. The resultant extract was re-dissolved with $10 \mathrm{~mL} \mathrm{H}_{2} \mathrm{O}$. The resulting solution was mixed with $600 \mathrm{mg}$ PVPP, left standing for $60 \mathrm{~min}$, and then centrifuged ( $16400 \mathrm{~g}, 4{ }^{\circ} \mathrm{C}, 10 \mathrm{~min}$ ). The resulting supernatant was loaded on an Amberlite XAD-2 column and eluted successively with $15 \mathrm{~mL}$ water, $15 \mathrm{~mL}$ pentane : dichloromethane $(2: 1)$, and $10 \mathrm{~mL}$ methanol. The methanol eluate was concentrated in a vacuum, dried under nitrogen gas, and derivatized with $70 \mu \mathrm{L}$ of MSTFA at $37^{\circ} \mathrm{C}$ for $60 \mathrm{~min}$. The MSTFA derivatives were analyzed by GC-MS equipped with an HP-5 column $(30 \mathrm{~m} \times 0.25 \mathrm{~mm} \times 0.25 \mu \mathrm{m}$, Agilent Technologies, Germany). The injector temperature was $240{ }^{\circ} \mathrm{C}$, the splitless mode was used with a splitless time of $1 \mathrm{~min}$, and helium was the carrier gas with a velocity of $1 \mathrm{~mL} \mathrm{~min}^{-1}$. The GC

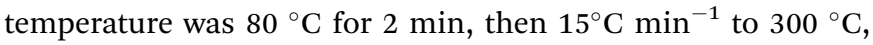
followed by a hold at $300{ }^{\circ} \mathrm{C}$ for $10 \mathrm{~min}$. The MS was operated in full-scan mode $(\mathrm{m} / \mathrm{z} 150-550)$. The authentic standards $(R)-1 \mathrm{PE}$ $\beta$-primeveroside and $(S)$-1PE $\beta$-primeveroside were obtained by chemical synthesis. ${ }^{\mathbf{1 4}}$

\section{Analysis of phytohormones}

To analyze the phytohormones, $1 \mathrm{~g}$ of finely powdered flowers was extracted with $10 \mathrm{~mL}$ of $80 \%$ methanol overnight. The supernatant was dried under nitrogen gas to remove methanol. The residual water was adjusted to $\mathrm{pH} 2.8$ with acetic acid and extracted with an equal volume of ethyl ether twice. The upper phase was combined and dried under nitrogen gas. The residual was dissolved in methanol, and phytohormones, including JA, ABA, and salicylic acid, were analyzed by an ultraperformance liquid chromatography/quadrupole time-of-flight mass spectrometry (UPLC-QTOF-MS) (Acquity UPLC I-Class/ Xevo® G2-XS QTOF, Waters Corporation, Milford, MA, USA) equipped with an AQUITY UPLC BEH C18 column (Waters Corporation, $2.1 \mathrm{~mm} \times 100 \mathrm{~mm} \times 1.7 \mu \mathrm{m})$. Solvent A was distilled water with $0.1 \%(\mathrm{v} / \mathrm{v})$ formic acid. Solvent B was methanol with $0.1 \%(\mathrm{v} / \mathrm{v})$ formic acid. Elution was started using $30 \%$ solvent B for $4 \mathrm{~min}$, then linearly increased to $65 \%$ within $15 \mathrm{~min}$. The flow rate was $0.25 \mathrm{~mL} \mathrm{~min}^{-1}$. The column temperature was $40{ }^{\circ} \mathrm{C}$. The MS conditions were capillary voltage: $2.5 \mathrm{kV}$; source temperature: $100{ }^{\circ} \mathrm{C}$; desolvation temperature: $350{ }^{\circ} \mathrm{C}$; cone gas flow: $50 \mathrm{~L} \mathrm{~h}^{-1}$; and desolvation gas flow: $600 \mathrm{~L} \mathrm{~h}^{-1}$.

\section{Transcript expression analysis of related genes}

RNA was isolated from $500 \mathrm{mg}$ flowers using a Quick RNA Isolation Kit (Huayueyang, Beijing, China). Then, $20 \mu \mathrm{L}$ of cDNA 
was synthesized from $500 \mathrm{ng}$ of total RNA using PrimeScript ${ }^{\mathrm{TM}}$ RT reagent Kit (Takara, Dalian, China) according to the manufacturer's instructions. The reverse transcription condition was $37{ }^{\circ} \mathrm{C}$ for $15 \mathrm{~min}$, followed by $85{ }^{\circ} \mathrm{C}$ for $5 \mathrm{~s}$. The primers used in the quantitative real-time PCR (qRT-PCR) are listed in Table S1. $\dagger$ Elongation factor- $1 \alpha(\mathrm{EF}-1 \alpha)$ was used as an internal reference. Reactions were performed using SYBR® PrimeScript ${ }^{\mathrm{TM}}$ RT-PCR Kit (Perfect Real Time) (Takara, Dalian, China) in a $20 \mu \mathrm{L}$ volume containing $10 \mu \mathrm{L}$ SYBR® Premix Ex Taq ${ }^{\mathrm{TM}}(2 \times), 0.4 \mu \mathrm{M}$ each of the specific sense and antisense primers, and $2 \mu \mathrm{L}$ of $50 \times$ diluted template. The qRT-PCR was carried out on a Roch LightCycler 480 under conditions of $95^{\circ} \mathrm{C}$ for $30 \mathrm{~s}$, followed by 40 cycles of $95{ }^{\circ} \mathrm{C}$ for $3 \mathrm{~s}$ and $60{ }^{\circ} \mathrm{C}$ for $34 \mathrm{~s}$. A melt curve was performed at the end of each reaction to verify PCR product specificity. The $2^{-\Delta \Delta C_{\mathrm{t}}}$ method was used to calculate the relative expression levels. ${ }^{17}$

\section{Statistical analysis}

Statistical analysis was performed using the SPSS Ver. 19.0 software. The paired and independent samples $t$-tests were used to determine differences between two groups. A probability level of $5 \%(p \leq 0.05)$ was considered significant.

\section{Acknowledgements}

This study was supported by the National Natural Science Foundation for Young Scholars of China (31500244), Guangdong Natural Science Foundation (2014A030310131 and 2016A030313652), the Guangdong Innovation Team of Modern Agricultural Industry Technology System (2016LM1143), and the "100 Talents Programme of the Chinese Academy of Sciences" (Y321011001 and 201209). We thank Prof. Xiaoli Tong at the South China Agricultural University for identification of insects.

\section{Notes and references}

1 F. Negre-Zakharov, M. C. Long and N. Dudareva, in PlantDerived Natural Products, ed. A. E. Osbourn and V. Lanzotti, Springer Berlin, Heidelberg, 2009, vol. 19, pp. 405-431.

2 E. Pichersky and J. Gershenzon, Curr. Opin. Plant Biol., 2002, 5, 237-243.

3 F. Dong, X. M. Fu, N. Watanabe, X. G. Su and Z. Y. Yang, Molecules, 2016, 21, 124, DOI: 10.3390/molecules21020124.

4 S. Allmann and I. T. Baldwin, Science, 2010, 329, 1075-1078. 5 F. Negre, C. M. Kish, J. Boatright, B. Underwood, K. Shibuya, C. Wagner, D. G. Clark and N. Dudareva, Plant Cell, 2003, 15, 2992-3006.

6 M. R. M. Neiland and C. C. Wilcock, Am. J. Bot., 1998, 85, 1657-1671.
7 R. R. Junker and N. Bluthgen, Evolutionary Ecology Research, 2008, 10, 295-308.

8 J. Kunze and A. Gumbert, Behav. Chem. Ecol., 2001, 12, 447456.

9 R. J. Gegear and T. M. Laverty, Anim. Behav., 2005, 69, 939949.

10 A. Balkenius and A. Kelber, Naturwissenschaften, 2006, 93, 255-258.

11 A. Shuttleworth and S. D. Johnson, Ann. Bot., 2009, 103, 715725.

12 N. B. Theis, M. Lerdau and R. A. Raguso, Int. J. Plant Sci., 2007, 168, 587-601.

13 F. Dong, Z. Y. Yang, S. Baldermann, Y. Kajitani, S. Ota, H. Kasuga, Y. Imazeki, T. Ohnishi and N. Watanabe, J. Plant Physiol., 2012, 169, 217-225.

14 Y. Zhou, F. Dong, A. Kunimasa, Y. Q. Zhang, S. H. Cheng, J. M. Lu, L. Zhang, A. Murata, F. Mayer, P. Fleischmann, N. Watanabe and Z. Y. Yang, J. Agric. Food Chem., 2014, 62, 8042-8050.

15 Y. Zhou, L. Zhang, J. D. Gui, F. Dong, S. H. Cheng, X. Mei, L. Y. Zhang, Y. Q. Li, X. G. Su, S. Baldermann, N. Watanabe and Z. Y. Yang, Plant Mol. Biol. Rep., 2015, 33, 253-263.

16 F. Dong, Y. Zhou, L. T. Zeng, Q. Y. Peng, L. Zhang, X. G. Su, N. Watanabe and Z. Y. Yang, Molecules, 2016, 21, 1106, DOI: 10.3390/molecules21091106.

17 K. J. Livak and T. D. Schmittgen, Methods, 2001, 25, 402-408. $18 \mathrm{H}$. E. M. Dobson, in Insect-Plant Interactions, ed. E. A. Bernays, CRC Press, Boston, 1994, vol. 5, pp. 46-81.

19 L. Tollsten, Nord J. Bot., 1993, 13, 495-499.

20 F. P. Schiestl and M. Ayasse, Oecologia, 2001, 126, 531-534.

21 J. D. Gui, X. M. Fu, Y. Zhou, T. Katsuno, X. Mei, R. F. Deng, X. L. Xu, L. Zhang, F. Dong, N. Watanabe and Z. Y. Yang, J. Agr. Food Chem., 2015, 63, 6905-6914.

22 S. Ohgami, E. Ono, M. Horikawa, J. Murata, K. Totsuka, H. Toyonaga, Y. Ohba, H. Dohra, T. Asai, K. Matsui, M. Mizutani, N. Watanabe and T. Ohnishi, Plant Physiol., 2015, 168, 464-477.

23 D. G. Clark, C. Richards, Z. Hilioti, S. LindIversen and K. Brown, Plant Mol. Biol., 1997, 34, 855-865.

24 J. Q. Wilkinson, M. B. Lanahan, D. G. Clark, A. B. Bleecker, C. Chang, E. M. Meyerowitz and H. J. Klee, Nat. Biotechnol., 1997, 15, 444-447.

25 M. Kato, H. Shimizu, T. Onozaki, N. Tanikawa, H. Ikeda, T. Hisamatsu and K. Ichimura, J. Jpn. Soc. Hortic. Sci., 2002, 71, 385-387.

26 K. Weterings, M. Pezzotti, M. Cornelissen and C. Mariani, Plant Physiol., 2002, 130, 1190-1200. 